\title{
INTERACTS (INTErventional Radiotherapy ACtive Teaching School) guidelines for quality assurance in choroidal melanoma interventional radiotherapy (brachytherapy) procedures
}

\author{
Luca Tagliaferri, MD, PhD', Monica Maria Pagliara, MD², Luca Boldrini, MD!, Carmela Grazia Caputo, MD², \\ Prof. Luigi Azario, MD³, Maura Campitelli, MD!, Prof. Maria Antonietta Gambacorta, MD, PhD!, \\ Prof. Daniela Smaniotto, MD! Vincenzo Frascino, MD', Francesco Deodato, MD, Prof. Alessio Giuseppe Morganti, MD5 , \\ Prof. György Kovács, MD, PhD 6,7, Prof. Vincenzo Valentini, MDl,8, Prof. Maria Antonietta Blasi, MD² \\ 'Gemelli Advanced Radiation Therapy Center, Fondazione Policlinico Universitario "A. Gemelli", Università Cattolica del Sacro Cuore, Rome, \\ Italy, 2Department of Ophthalmology, Fondazione Policlinico Universitario "A. Gemelli", Università Cattolica del Sacro Cuore, Rome, Italy, \\ ${ }^{3}$ Physics Institute, Fondazione Policlinico Universitario "A. Gemelli", Università Cattolica del Sacro Cuore, Rome, Italy. "Department of Radiation \\ Oncology, Fondazione di Ricerca e Cura Giovanni Paolo II, Università Cattolica del Sacro Cuore, Campobasso, Italy, ${ }^{5}$ Division of Radiation \\ Oncology, S. Orsola Malpighi Hospital, University of Bologna, Italy, ${ }^{9}$ Interdisciplinary Brachytherapy Unit, University of Lübeck/UKSH CL, \\ Lübeck, Germany, ${ }^{7}$ INTERACTS (Interventional Radiotherapy Active Teaching School) Educational Program Director, ${ }^{8}$ INTERACTS (Interventional \\ Radiotherapy Active Teaching School) School Director
}

\begin{abstract}
Eye plaque brachytherapy represents a safe and effective therapeutic approach for choroidal melanoma, combining clinical outcomes with an eye and visual preservation.

As it represents a complex procedure, a specific quality assurance program is strongly suggested to improve patients and operators safety, and to reduce possible complications linked to surgical procedure and radiation exposure.

The aim of this paper is to describe the INTERACTS (Interventional Radiotherapy Active Teaching School) guidelines for quality assurance in choroidal melanoma interventional radiotherapy (brachytherapy) used in our institution.

J Contemp Brachytherapy 2017; 9, 3: 287-295

DOI: https://doi.org/10.5114/jcb.2017.6876l
\end{abstract}

Key words: brachytherapy, choroidal melanoma, ocular, medical education.

\section{Purpose}

Ocular melanoma represents the second most common type of melanoma after cutaneous form. It generally originates from uvea ( $82.5 \%$ of the cases), and is the first primary intraocular malignancy with an incidence that varies between 2 cases per million in the Southern Europe and 8 per million in the Northern Europe [1,2]. For its peculiar early clinical presentation (scotomas accompanied or not by pain, eye pressure modifications), it is generally diagnosed in early stage; however, even disease dissemination is not uncommon (5\% of cases) presenting hepatic metastases close to $90 \%$ [3]. Radiation therapy has gained a major role in the treatment of this disease as described by the COMS (Collaborative Ocular Melanoma Study), which showed no survival differences between patients whose tumors were treated with ${ }^{125} \mathrm{I}$ eye plaque brachytherapy and those treated with surgical enucleation; however, radiotherapy offers improved visual preservation and cosmetic outcome [4]. Further experiences have confirmed these observations also by the use of different radionuclides $\left({ }^{106} \mathrm{Ru} / \mathrm{Rh}\right.$ or $\left.{ }^{103} \mathrm{Pd}\right)[5,6,7]$. As the size and location of disease have a crucial role in the choice of the clinical management of this tumor, and several aspects should be taken into account, a multidisciplinary approach is strongly suggested $[7,8,9,10]$. Eye brachytherapy represents therefore a complex procedure that involves a skilled multidisciplinary and multiprofessional team, and requires strict quality assurance (QA) protocols to assure optimal clinical results and operator safety. Our Institution has developed an internal QA handbook about radiation therapy treatments in 2001, with several chapters dealing with the different aspects of the radiation oncology workflow [11,12]. A specific 
chapter for eye plaque brachytherapy procedures has been added in 2006, including the detailed description of treatment procedures (before, during, and after), and treatment related documentation (forms) as well the necessary checklists. The first principle of the QA procedures is their 'traceability', which is the possibility to identify, through the signature of specific reports, the operator who has realized their single steps.

The second one is the 'verifiability' of the performed procedures that is based on the use of quality indicators, demonstrating the adherence to predefined QA standards.

Recently, we have proposed eye brachytherapy QA guidelines in the frame of the Interventional Radiotherapy Active Teaching School (INTERACTS), which has its mission in the education and research in interventional radiotherapy [13].

The aim of this paper is to describe the INTERACTS guidelines for QA in choroidal melanoma interventional radiotherapy (brachytherapy) workflow, which is articulated in five main issues (Figure 1).

\section{Step 1. Multidisciplinary tumor board: a case presentation and treatment procedure decision}

The published American Brachytherapy Society (ABS) consensus guideline for eye plaque brachytherapy recommends to refer choroidal melanoma patients to experienced sub-specialty centers with high amount

\begin{tabular}{|l|}
\hline $\begin{array}{l}\text { 1. Multidisciplinary tumor board: case presentation } \\
\text { and treatment choice }\end{array}$ \\
\hline 2. Treatment planning: plan calculation and pre-plan approval \\
\hline 3. Source preparation: applicator loading and sterilization \\
\hline 4. Surgery: Plaque implantation \\
\hline 5. Treatment and plaque removal \\
\hline
\end{tabular}

Fig. 1. Eye plaque brachytherapy workflow main steps of treated patients per year, as differences in procedural volume have been reported as a significant predictors of risk adjusted mortality $[7,8,9]$.

Our Institution treats on average 40 choroidal melanoma cases per year, and represents a national reference center for this treatment. From December 2006 to December 2016, we have treated 417 choroidal melanoma patients with the use of ${ }^{106} \mathrm{Ru} / \mathrm{Rh}$ (343 cases) plaques and ${ }^{125}$ I (74 cases) treatments. Since the first eye plaque implant, an internally developed QA protocol has always been followed, and no major clinical relevant incidents have ever been registered.

\section{Multidisciplinary tumor board}

The clinical practice workflow includes the presentation of the cases in a weekly multidisciplinary tumor board (MTB), which includes an ophthalmologist skilled in plaque placement surgery, as well as a radiation oncologist and a physicist familiar with interventional radiotherapy applications $[7,8,9]$. A medical oncologist is also involved in the discussion in case of metastatic presentation of disease. When computed tomography (CT) or magnetic resonance imaging (MRI) are available, images are discussed with a radiologist (Table 1).

Multidisciplinary tumor boards allow for more effective control of patient's workflow, optimizing the role of the different figures involved and assuring a more accurate and complete disease staging and therapy indication with initial evidence of improvements in clinical outcome [10]. The ophthalmologist presents the patient's history and records to the other MTB members. General physical exam, visual acuity and eye pressure, slit lamp and ophthalmoscopy, ultrasound (US) A and B-scans, angiography, and wide field fundus photography findings are reported and discussed [7,14,15,16,17,18,19,20,21,22,23]. Histopathological data are not mandatory and biopsy can be substituted with a clinical diagnosis by an ophthalmologist skilled in eye melanoma, based on different exams with a false positive rate below 1-2\% for expert personnel [24].

\section{Gross tumor volume (GTV) definition}

The ophthalmologist provides a detailed diagram of fundus oculi with tumor margins orientation; the distance from optic nerve and fovea are reported. Basal di-

Table 1. Multidisciplinary tumor board composition

\begin{tabular}{lcc} 
Multidisciplinary team member & Presence & Role \\
\hline Ophthalmologist & Always & $\begin{array}{c}\text { Introduces patient's and lesion's characteristics and defines the } \\
\text { gross tumor volume (GTV) }\end{array}$ \\
\hline Radiation oncologist & Always & $\begin{array}{c}\text { Confirms the treatment indication to eye brachytherapy, defines } \\
\text { the clinical target volume (CTV) and planning target volume (PTV), } \\
\text { chooses the most appropriate radionuclide along with physicist, } \\
\text { and prescribes the dose to the apex }\end{array}$ \\
\hline Physicist & Always & $\begin{array}{c}\text { Performs treatment planning, and is involved in radionuclide } \\
\text { choice }\end{array}$ \\
\hline Medical oncologist & Only in some cases & Discussion in case of metastatic presentation of disease \\
\hline Radiologist & Only in some cases & Discussion in case of CT or MRI availability
\end{tabular}


ameters and apex height of the disease are also carefully measured for gross tumor volume (GTV) definition. Ultrasound scans have a crucial role in disease extension measurement, and for a reliable evaluation of the GTV, the use of both A and B-scans is strongly recommended. Tumor diameters are defined with B-scans while disease height assessment is evaluated with A-scans, and their information cannot be replaced by other US techniques, even if with higher nominal resolution [14].

\section{Interventional radiotherapy (brachytherapy) indication}

For patients with melanomas $>5 \mathrm{~mm}$ apex distance, a systemic staging is usually requested.

We consider patients eligible for primary eye brachytherapy if choroidal melanoma average dimensions (thickness, 2-9 mm) and maximum diameters $<16 \mathrm{~mm}$ are present without systemic disease. The treatment of diseases that do not meet these parameters are to be discussed in detail by the MDT to consider the possibility to integrate therapeutic and palliative purposes of the treatment without excluding a priori brachytherapy. The reasons of each decision are documented in details in the patient's chart.

In case of systemic diseases or in ocular metastases, we consider eye brachytherapy as a palliative treatment, in order to prevent eye pain due to tumor caused secondary glaucoma.

\section{Clinical target volume definition}

The radiation oncologist then confirms the treatment indication for eye brachytherapy. The radiation oncologist defines the clinical target volume (CTV) and prescribes the dose to the tumor apex. This volume represents a safety margin extension of 1-2 $\mathrm{mm}$ in all directions, accounting for microscopic disease spread along uvea and sclera.
The CTV thickness is defined taking into account the tumor thickness, usually read on B-scan sonography images with a $1 \mathrm{~mm}$ extension for the sclera. A case by case defined planning target volume (PTV), extra safety margin can be added by the radiation oncologist in case of uncertainties in tumor delineation and/or plaque localization $[24,25]$.

\section{Radionuclide definition and dose prescription}

Considering the aforementioned dimensions, the radiation oncologist along with the physicist defines the most appropriate radionuclide for plaque loading. In our Institution, we use ${ }^{106} \mathrm{Ru} / \mathrm{Rh}$ plaques for lesions less than $5 \mathrm{~mm}$ in thickness, as they allow more simple radioprotection procedures with better toxicity profile (especially in terms of cataract and retinopathy), and overall survival and local control outcomes comparable to ${ }^{125}$ I seeds, which are generally used for thicker lesions [26,27].

The prescription dose suggested by the GEC ESTRO is $100 \mathrm{~Gy}$ to the apex for ${ }^{106} \mathrm{Ru} / \mathrm{Rh}$, and $70-150 \mathrm{~Gy}$ to the apex for ${ }^{125} \mathrm{I}$ with dose rates between $50-100 \mathrm{cGy} / \mathrm{h}$, while the American Brachytherapy Society suggests a prescription dose of 70-100 Gy to the apex with a dose rate not less than $60 \mathrm{cGy} / \mathrm{h}$ for both isotopes [24].

In our Institution, the dose is prescribed to the apex with a total prescription of $100 \mathrm{~Gy}$ for ${ }^{106} \mathrm{Ru} / \mathrm{Rh}$, and $85 \mathrm{~Gy}$ for ${ }^{125} \mathrm{I}$ with dose rate $>50 \mathrm{cGy} / \mathrm{h}$.

If consensus is reached on the eye brachytherapy indication and isotope choice, the multidisciplinary group proceeds to phase 2 : treatment planning.

\section{Step 2. Treatment planning: plan calculation and pre-plan approval}

The physicist calculates a provisional treatment plan (pre-plan) using a dedicated 3D calculation software (Plaque Simulator ${ }^{\mathrm{TM}}$, Bebig, Germany), and performing

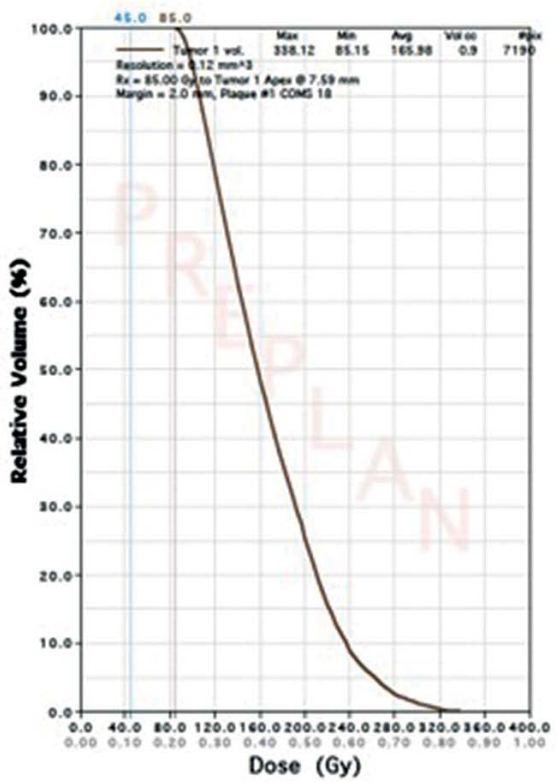

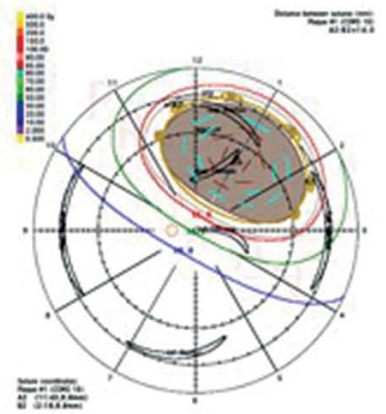

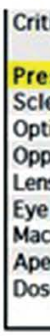

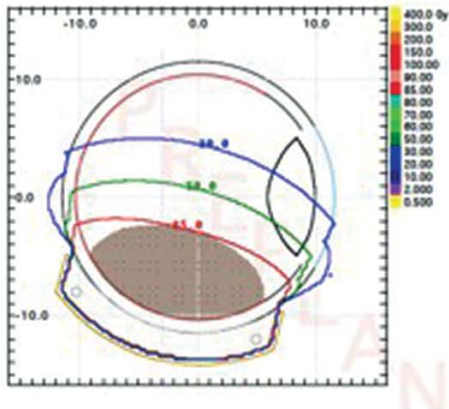

Dist. from plaque ( $\mathrm{mm}$ ) 8.590 Tumor 1 Apex $97.59 \mathrm{~mm}$ 1.000 (from plaque center) 15.05 (center to center) 22.00 (from plaque center) 15.85 (center to center) 11.50 (from plaque center)

13.44 (center to center)

8.590 (from plaque center)

Fig. 2. Example of 3D treatment planning (Plaque Simulator ${ }^{\mathrm{TM}}$, Bebig, Germany) 
Table 2. Planning tips for radionuclides used in our Institution

\begin{tabular}{lccc} 
Source & Prescription doses & Treatment planning constraints & Plan acceptance criteria \\
\hline${ }^{106} \mathrm{Ru} / \mathrm{Rh}$ & $100 \mathrm{~Gy}(>50 \mathrm{cGy} / \mathrm{h})$ & Sclera $<1000 \mathrm{~Gy}$ & $>95 \%$ target coverage \\
\hline${ }^{125} \mathrm{I}$ & $85 \mathrm{~Gy}(>50 \mathrm{cGy} / \mathrm{h})$ & Sclera $<1000 \mathrm{~Gy}$ & $>95 \%$ target coverage
\end{tabular}

an independent check of treatment time by manual calculation.

Plaque planning provides a graphical distribution of dose, dose volume histogram (DVH) of the therapy volumes (target and organs at risk), and dose rate information (Figure 2).

Plaque arrangements can be different varying radionuclides, source strengths, and ring dimensions [28].

In our Institution, plans are approved when at least $95 \%$ of target volume is covered by the prescribed dose. An indicative maximal constraint of $1000 \mathrm{~Gy}$ is considered for the sclera [24] (Table 2).

The main advantage of 3D planning is represented by the possibility to identify eventual peripheral underdosed target areas that do not allow plan delivery: in these cases, patients could be addressed to adjuvant transpupillary thermotherapy (TTT) or photodynamic therapy (PDT) [29,30].

\section{Independent check}

Our QA protocol requires an independent check of the treatment workflow at this level.

The internal guidelines used in our Institution define the independent check as the revision of the completeness and accuracy of accomplished procedures performed by an operator with adequate professional skills, who has not been directly involved in the treatment process [31]. Main aim of the independent check process is to guarantee the correct flow of treatment procedures. A physicist proceeds with the independent check of the dosimetrical and geometrical parameters of the pre-plan, and a radi- ation oncologist performs a medical independent check verifying all therapy passages and gives his authorization for the treatment to proceed. The independent check is done following a check list comprehending the verifications of several parameters: patient name, ocular US report, therapy volume measurement (GTV-CTV-PTV), implant notes, correspondence between US findings and treatment plan, plaque code, correspondence between the prescribed and the planned dose, and presence of physics independent check.

\section{Informed consent}

At this time, the total treatment time is calculated: surgery and patient admission to the wards are scheduled accordingly. A double copy of patient's informed consent is then collected: one copy is to be maintained in brachytherapy ward records, while the second one is to be stored in ophthalmology archives. Informed consent is collected by ophthalmologist and radiation oncologist at the same time.

\section{Step 3. Source preparation: applicator loading and sterilization}

After the pre-plan approval and following the independent checks, radiation therapy technician (RTT) proceeds with the preparation of the chosen ${ }^{106} \mathrm{Ru} / \mathrm{Rh}$ plaque or arranges the selected seeds configuration for ${ }^{125}$ I treatment. Following the pre-treatment plan indication, the RTT places the seeds inside a silicone support and covers with a protection plaque (Figure 3 ).
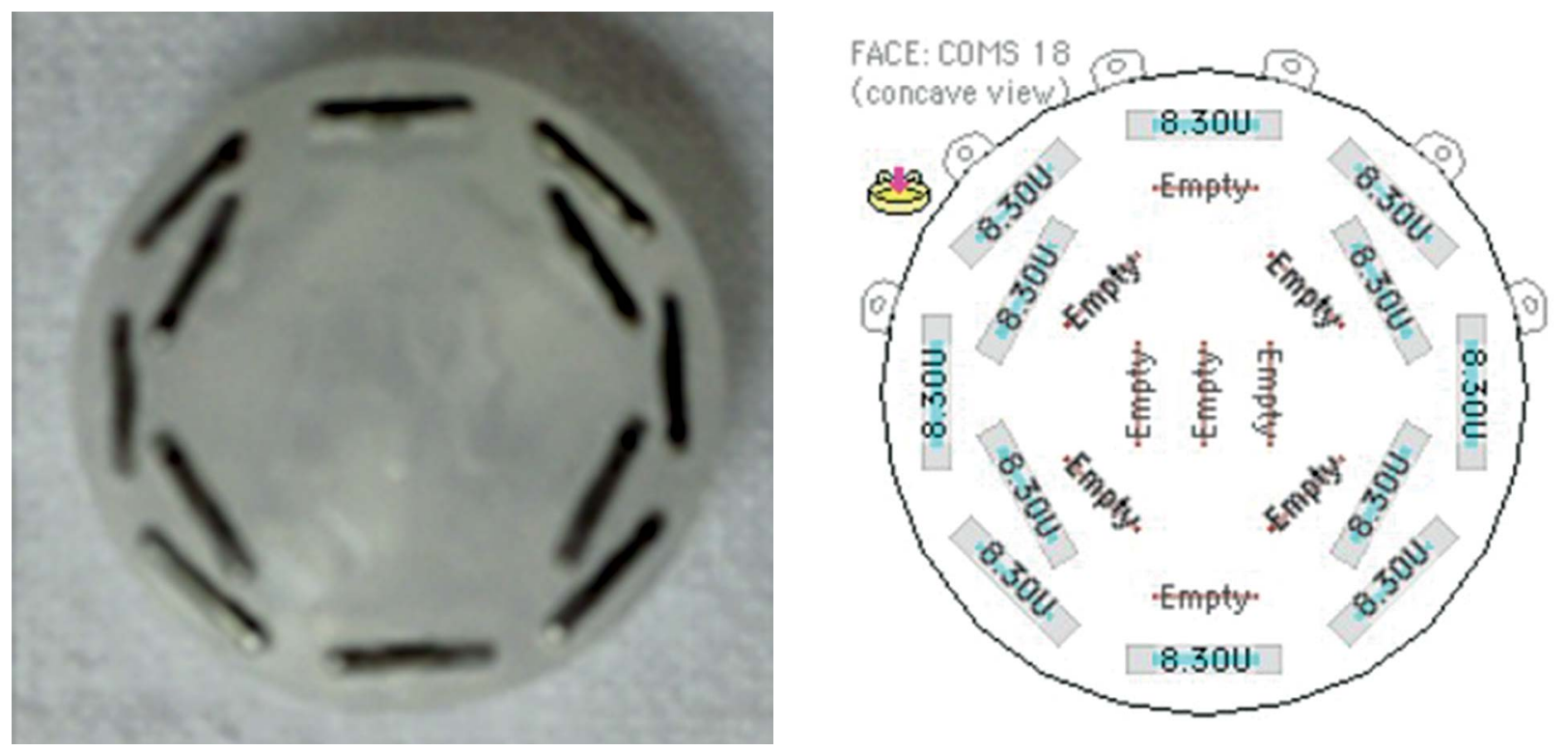

Fig. 3. Seeds configuration in the silicone support (left) following pre-treatment plan indications (right) 

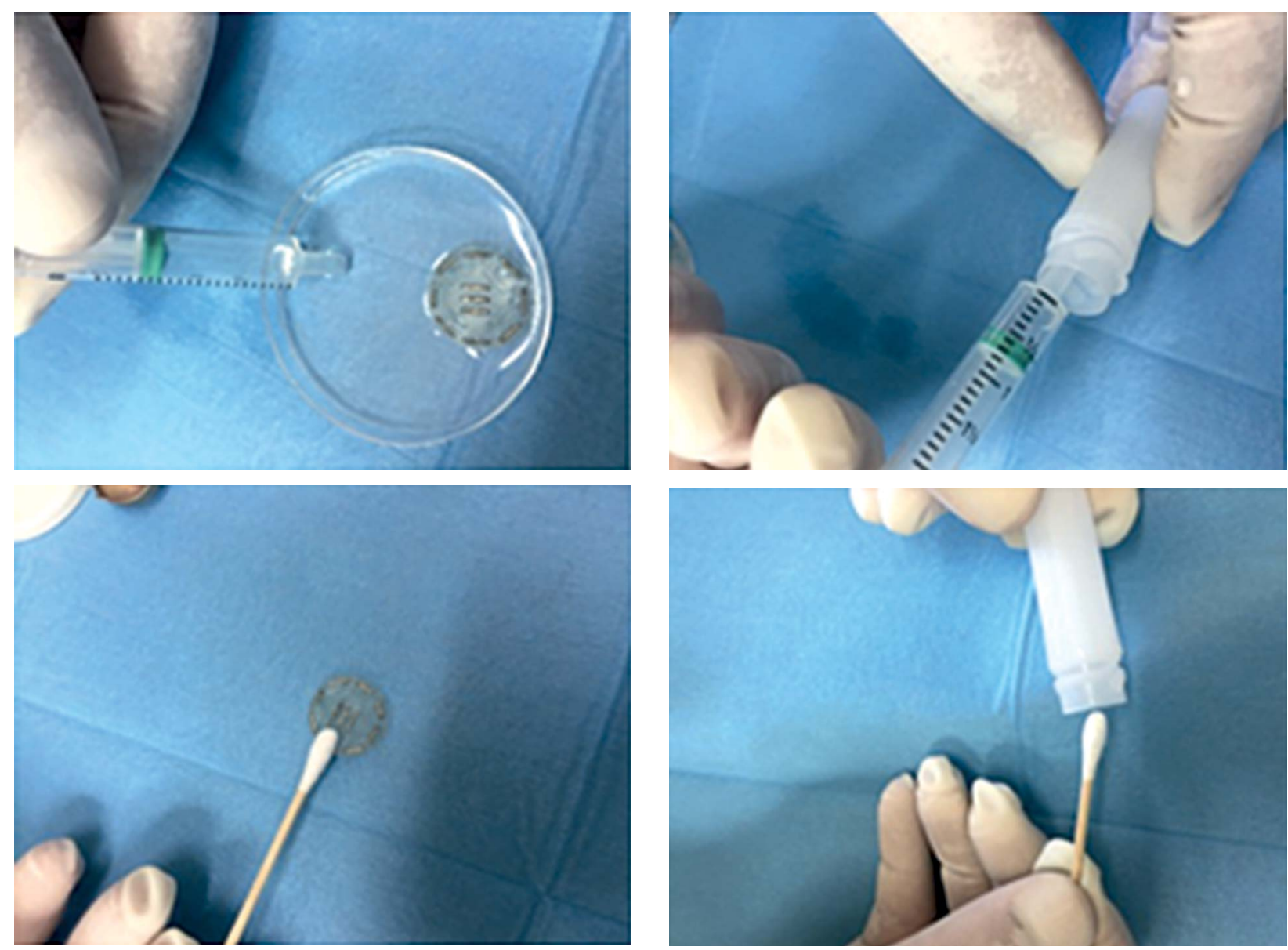

Fig. 4. ${ }^{125}$ I source contamination check with water (top) and swab (bottom) test

The manipulation of the seeds requires particular attention, and specific rules need to be followed in order to avoid seed damage as operating errors can cause a possible contamination [32]. Our internal QA protocol suggests therefore not to clamp the seed too strongly during its manipulation, as these operations can damage its surface. A contamination check is mandatory before treatment and it can be performed in two ways: a water test (immersing the plaque in water) and with the smear test (gently brushing the applicator with a cotton swab). Both water and swab are then checked for contamination to confirm the absence of leaking seeds (Figure 4).

Thanks to this procedure, in our experience, one treatment out of 74 had to be re-planned due to inadequate source preparation with consequent contamination (error detected by contamination test).

The plaques then undergo the sterilization process (autoclave for ${ }^{106} \mathrm{Ru} / \mathrm{Rh}$ plaques and gas plasma for ${ }^{125} \mathrm{I}$ ). During this process, the pressure and temperature could temporarily modify the silicone insert, so that the seeds can move from the original position (Figure 5).

For this reason, the seeds configuration needs to always be checked after the sterilization process and before the surgical implant. In our experience, we detected one case of seeds dislodgment after sterilization out of 74 performed procedures. After this incident, we have modified the QA procedures and introduced the fixation of

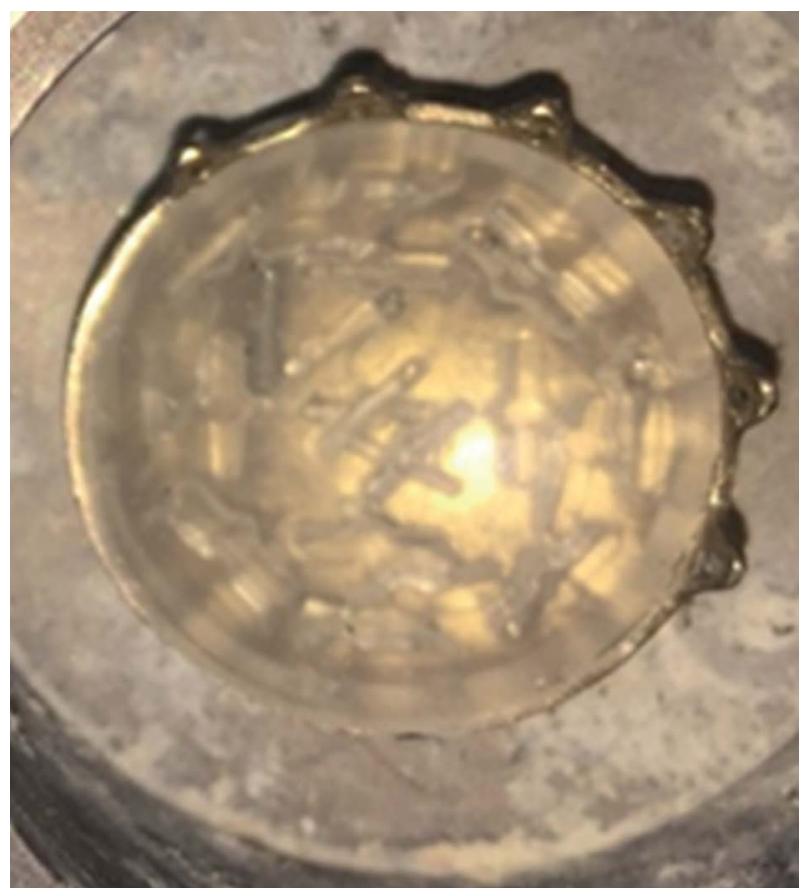

Fig. 5. During the sterilization process, the pressure and temperature can temporarily modify the silicone insert, so that the seeds can move away from the original position. Example of seeds dislodgment after sterilization 

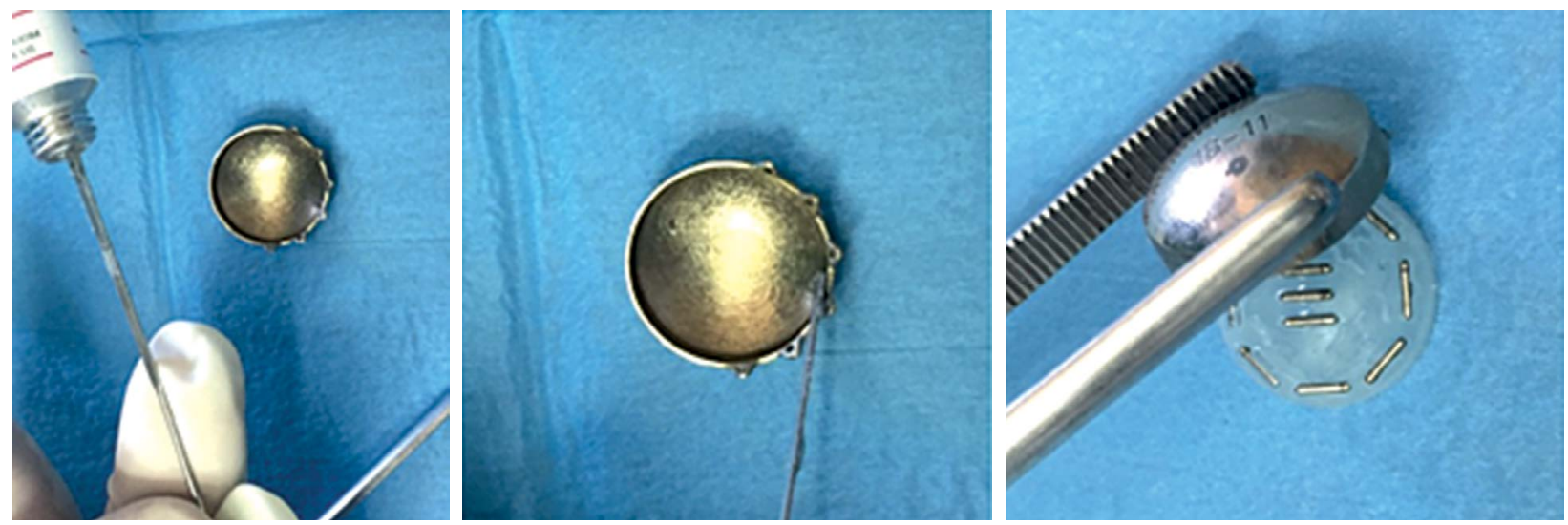

Fig. 6. Procedure to reduce the risk of seeds dislodgment after sterilization: a proper amount of a glue is used to fix the silicone support to the plaque before sterilization

the silicone support to the plaque before sterilization with a proper amount of a glue (Figure 6). After introduction of this procedure, no errors in the configuration of seeds after sterilization have been observed.

Following the QA procedures, the applicator is ready for surgical implantation.

\section{Step 4. Surgery: plaque implantation}

The implantation of the plaque is performed by ophthalmologist together with radiation oncologist, generally in local anesthesia.

\section{Surgical technique}

The surgical approach consists of a conjunctival peritomy with disease quadrant exposure (Figure 7A) and localization of tumor (GTV) borders by transillumination of the eye, by placing an optic fiber light at 180 degrees from the tumor [24] (Figure 7B). Then, ophthalmologist proceeds with the tumor border marking (using a sterile marking pen) and adding a safety margin of 2-3 $\mathrm{mm}$ (Figure 7C). Considering this so defined area, a sterile dummy applicator of a proper size and shape is positioned and fixed with loose sutures to the overlying sclera (Figure 7D). These procedures contribute in minimizing the exposure of the personnel present in the operating theatre.

When the reliability of the implantation (tumor coverage) will be confirmed (even by transillumination photography and/or through US if necessary), the dummy will be replaced by the loaded applicator and the scleral sutures have to be tightened (Figures 7E and 7F).

\section{Surgical approach recommendations}

The use of US positioning confirmation is strongly suggested, especially for posterior lesions, as recent experiences show a relative local failure rate of $0 \%$ when it is performed [33]. During surgical procedures, the plaque needs to be manipulated by following specific rules. An incorrect handling of ${ }^{125} \mathrm{I}$ plaque can cause a displacement of the seeds: in our experience, this incident occurred once during procedure. After this episode, strict rules were introduced regarding plaque handling; we advise to manipulate it from peripheral lugs on the shell while avoiding touching the silicone insert. No other displacement incidents have been detected since the introduction of this procedure.

The risk of contamination is present also for ${ }^{106} \mathrm{Ru} / \mathrm{Rh}$ plaques, as they could be scratched during positioning. Our QA program suggests to use normal clamps to hold the plaque from lugs, and to prefer clamps covered by plastic material to touch the plaque in the operating theatre.

This recommendation is active in our institution since the first implant, and no contamination has ever been detected.

\section{Post-surgery procedures}

The exact positioning time of the plaque is recorded and communicated to physicist for treatment time confirmation and removal time calculation.

The patient is then transported to the ward with appropriate protection shell on the treated eye, lying in a bed pulled from the foot side (to maximize the distance between the source and the stretcher bearer). Elevators and corridors of the scheduled itinerary are left free in advance to avoid people accidental irradiation.

\section{Step 5. Treatment period and plaque removal}

Patients undergoing eye plaque brachytherapy with ${ }^{106} \mathrm{Ru} / \mathrm{Rh}$ are admitted to ophthalmology ward, while patients implanted with ${ }^{125} \mathrm{I}$ are admitted in the radio-protected interventional radiotherapy ward, in which a radiation shielded single room is available. In both cases, an appropriate support therapy is prescribed by ophthalmologist and by radiation oncologist. During the hospitalization, patient undergoes three visits per day, and general conditions (e.g. vital parameters or pain) are registered. Visitors are not allowed in the protected brachytherapy ward and the patient is requested to wear a protection shell on the eye when the personnel enters in the room.

The typical duration of an implant in situ varies between 1 and 7 days [34]. At the end of the calculated treatment time, the plaque is removed with a new surgical procedure: ${ }^{106} \mathrm{Ru} / \mathrm{Rh}$ plaques' integrity and ${ }^{125} \mathrm{I}$ seeds number and status are checked. Following the plaque re- 

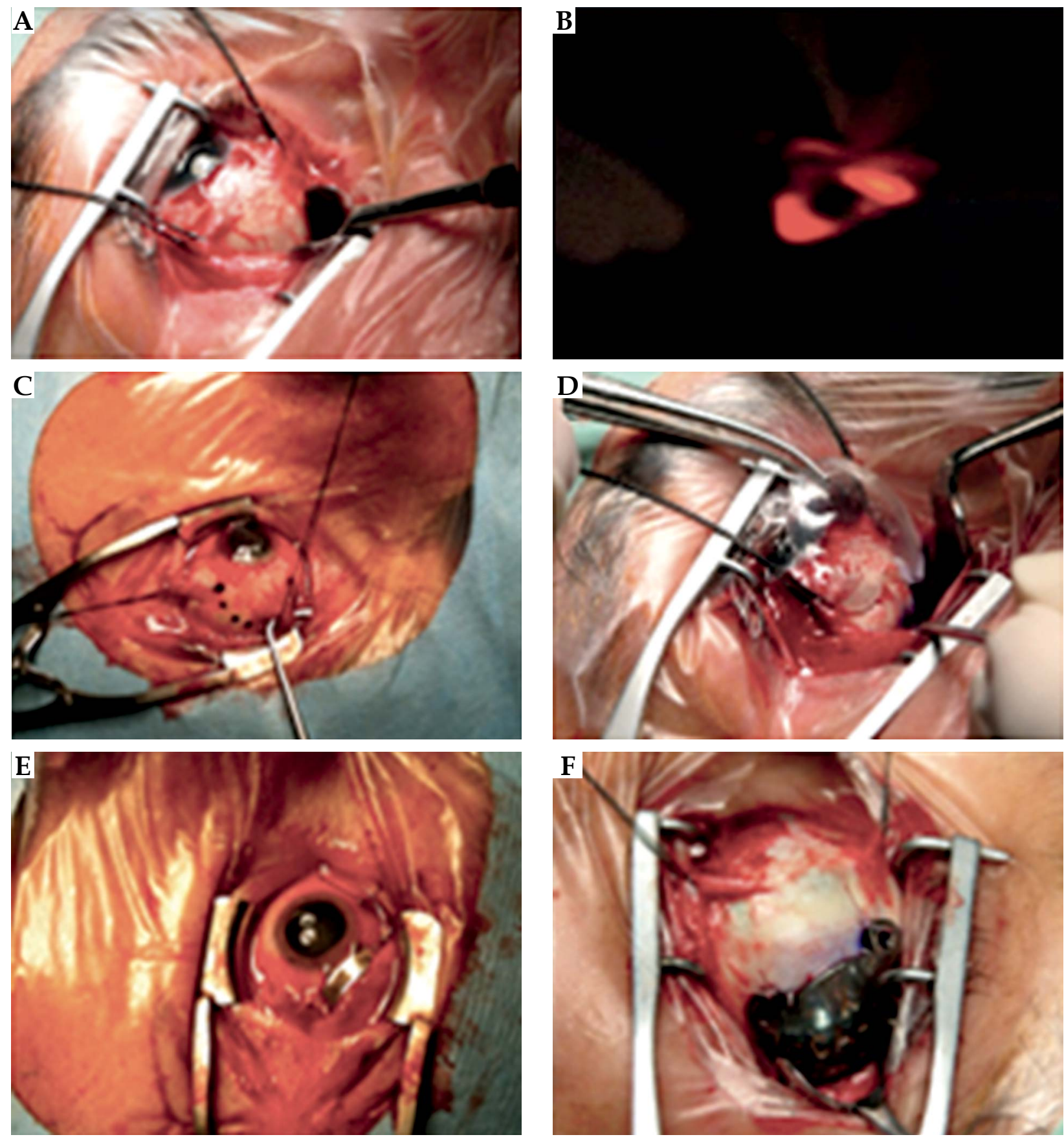

Fig. 7. The surgical phases as presented in the text

moval, a contamination check of the plaque by smear test and of patient's orbit is always performed (Figure 8).

The patient is then discharged with scheduled follow-up visit dates, and is asked to fill out a satisfaction questionnaire about overall treatment.

\section{Advantages and indicators of the INTERACTS QA guidelines}

The main advantages linked to the implementation of the INTERACTS QA guidelines in choroidal melanoma interventional radiotherapy are represented by the facilitation of the management of high amount of patients for such a complex procedure that requires a well-structured workflow, which combines surgical and radiotherapeutic skills.

The use of these QA guidelines could furthermore reduce legal arguments and medico-legal issues, thanks to the aforementioned principles of traceability and verifiability of single steps. The traceability of all procedures is granted by the use of QA modules, in which all steps are described in detail and involved personnel is clearly 

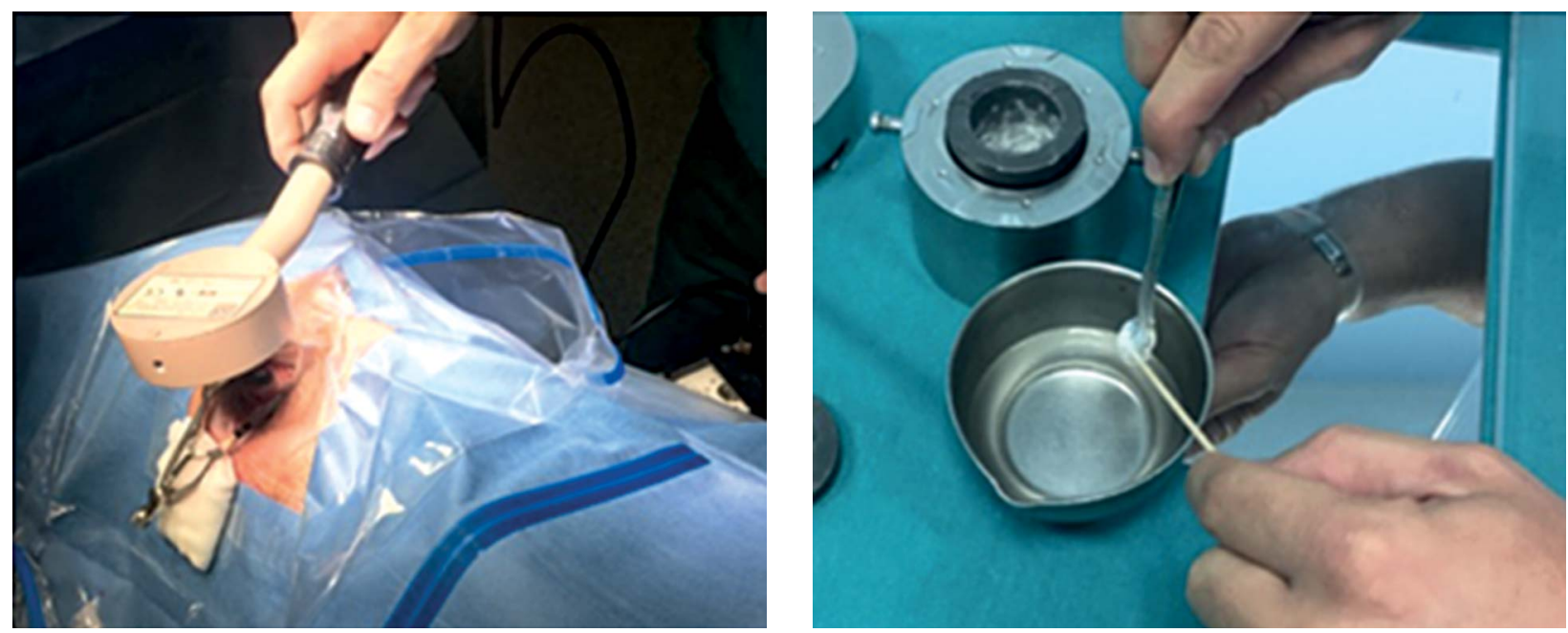

Fig. 8. Contamination check after brachytherapy treatment with orbit measurement (left) and plaque's smear test (right)

defined. Each of the involved figure places a signature in the specific field of dedicated form, once a step has been successfully accomplished.

In this context, the main quality indicators can be identified in: 1. Planned independent checks (medical and physical); 2. Contamination tests.

A third advantage of the application of these guidelines is related to their educational role, helping residents and in training personnel in optimizing their knowledge about the procedure and professional skills, imparting the importance to always operate as per QA frame.

Furthermore, eye melanoma is an uncommon disease: a multicentric data collection and interdisciplinary electronic database could therefore offer an efficient tool to produce valuable evidences and improve QA procedures through data sharing among subspecialty centers $[35,36]$.

\section{Conclusions}

Eye plaque brachytherapy is a highly specialized therapy that needs particularly trained personnel in experienced high workload centers. Due to the numerous figures involved in this kind of treatment, an accurate coordination between team members is required. Quality assurance protocols are strongly suggested to monitor the workflow, prevent accidents, and avoid complications. The adoption of the proposed INTERACTS guidelines for QA in choroidal melanoma interventional radiotherapy (brachytherapy) is therefore strongly suggested for eye brachytherapy treatments, as it offers high level of patient and operator safety as well as best possible clinical outcomes with a negligible rate of clinically relevant complications.

\section{Disclosure}

Authors report no conflict of interest.

\section{References}

1. Jovanovic P, Mihajlovic M, Djordjevic-Jocic J et al. Ocular melanoma: an overview of the current status. Int J Clin Exp Pathol 2013; 6: 1230-1244.
2. Virgili G, Gatta G, Ciccolallo L et al. Incidence of uveal melanoma in Europe. Ophthalmology 2007; 114: 2309-2315.

3. Diener-West M, Reynolds SM, Agugliaro DJ et al. Development of metastatic disease after enrollment in the COMS trials for treatment of choroidal melanoma: collaborative ocular melanoma study group report No 26. Arch Ophthalmol 2005; 123: 1639-1643.

4. Collaborative Ocular Melanoma Study Group. The COMS randomized trial of iodine 125 brachytherapy for choroidal melanoma: V. Twelve-year mortality rates and prognostic factors: COMS report No 28. Arch Ophthalmol 2006; 124: 1684-1693.

5. Diener-West M, Earle JD, Fine SL et al. The COMS randomized trial of iodine 125 brachytherapy for choroidal melanoma, iii: Initial mortality findings. COMS report No 18. Arch Ophthalmol 2001; 119: 969-982.

6. Sieving PA. Fifteen years of work: The COMS outcomes for medium-sized choroidal melanoma. Arch Ophthalmol 2001; 119: 1067-1068.

7. The American Brachytherapy Society consensus guidelines for plaque brachytherapy of uveal melanoma and retinoblastoma. American Brachytherapy Society - Ophthalmic Oncology Task. Brachytherapy 2014; 13: 1-14.

8. El Saghir NS, Keating NL, Carlson RW et al. Tumor boards: optimizing the structure and improving efficiency of multidisciplinary management of patients with cancer worldwide. Am Soc Clin Oncol Educ Book 2014; e461-466.

9. Osborne NH, Ghaferi AA, Nicholas LH et al. Evaluating popular media and internet-based hospital quality ratings for cancer surgery. Arch Surg 2011; 146: 600-604.

10. Pillay B, Wootten AC, Crowe H et al. The impact of multidisciplinary team meetings on patient assessment, management and outcomes in oncology settings: A systematic review of the literature. Cancer Treat Rev 2016; 42: 56-72.

11. Morganti AG, Smaniotto D, Luzi S et al. Quality handbook in radiotherapy. Brachytherapy: personal experience. Rays 2001; 26: 215-218.

12. Valentini V, Piermattei A, Marchetti M et al. Quality assurance in radiotherapy: personal experience. Rays 2001; 26: 209-212.

13. http://www.gemelliart.it/interacts.

14. Finger PT, Reddy S, Chin K. High-frequency ultrasound characteristics of 24 iris and iridociliary melanomas: Before and after plaque brachytherapy. Arch Ophthalmol 2007; 125: 1051-1058. 
15. Romani A, Baldeschi L, Genovesi-Ebert F et al. Sensitivity and specificity of ultrasonography, fluorescein videoangiography, indocyanine green videoangiography, magnetic resonance and radioimmunoscintigraphy in the diagnosis of primary choroidal malignant melanoma. Ophthalmologica 1998; 212: 44-46.

16. Finger PT, Garcia JP Jr, Pro MJ et al. "C-scan” ultrasound imaging of optic nerve extension of retinoblastoma. Br J Ophthalmol 2005; 89: 1225-1226.

17. Kook D, Kreutzer TC, Wolf A et al. Variability of standardized echographic ultrasound using $10 \mathrm{mHz}$ and high-resolution $20 \mathrm{mHz}$ B scan in measuring intraocular melanoma. Clin Ophthalmol 2011; 5: 477-482.

18. Marigo FA, Finger PT, McCormick SA et al. Iris and ciliary body melanomas: Ultrasound biomicroscopy with histopathologic correlation. Arch Ophthalmol 2000; 118: 1515-1521.

19. Chin K, Finger PT. Autofluorescence characteristics of suspicious choroidal nevi. Optometry 2009; 80: 126-130.

20. Freton A, Chin KJ, Raut R et al. Initial PET/CT staging for choroidal melanoma: AJCC correlation and second nonocular primaries in 333 patients. Eur J Ophthalmol 2012; 22: 236-243.

21. Shields CL, Kaliki S, Rojanaporn D et al. Enhanced depth imaging optical coherence tomography of small choroidal melanoma: Comparison with choroidal nevus. Arch Ophthalmol 2012; 130: 850-856.

22. Lommatzsch PK, Ballin RE, Helm W. Fluorescein angiography in the follow-up study of choroidal melanoma after 106Ru/106Rh plaque therapy. Retina 1987; 7: 148-155.

23. Rootman DB, Gonzalez E, Mallipatna A et al. Hand-held high resolution spectral domain optical coherence tomography in retinoblastoma: Clinical and morphologic considerations. $\mathrm{Br}$ J Ophthalmol 2013; 97: 59-65.

24. Gerbaulet A, Pötter R, Mazeron JJ et al. (eds.). The GEC ESTRO Handbook of Brachytherapy. ESTRO, Brussels 2002.

25. Gagne NL, Rivard MJ. Quantifying the dosimetric influences of radiation coverage and brachytherapy implant placement uncertainty on eye plaque size selection. Brachytherapy 2013; 12: 508-520.

26. Takiar V, Voong KR , Gombos DS, et al. A choice of radionuclide: Comparative outcomes and toxicity of ruthenium-106 and iodine-125 in the definitive treatment of uveal melanoma. Pract Radiat Oncol 2015; 5: e169-176.

27. Naseripour M, Jaberi R, Sedaghat A et al. Ruthenium-106 brachytherapy for thick uveal melanoma: reappraisal of apex and base dose radiation and dose rate. J Contemp Brachytherapy 2016; 8: 66-73.

28. Gagne NL, Cutright DR, Rivard MJ. Keeping an eye on the ring: COMS plaque loading optimization for improved dose conformity and homogeneity. J Contemp Brachytherapy 2012; 4: 165-175.

29. Tarlan B, Kiratlı H. Uveal Melanoma: Current Trends in Diagnosis and Management. Turk J Ophthalmol 2016; 46: 123-137.

30. Blasi MA, Laguardia M, Tagliaferri L et al. Brachytherapy alone or with neoadjuvant photodynamic therapy for amelanotic choroidal melanoma: functional outcomes and local tumor control. Retina 2016; 36: 2205-2212.

31. Valentini V, Glimelius B, Frascino V. Quality assurance and quality control for radiotherapy/medical oncology in Europe: guideline development and implementation. Eur J Surg Oncol 2013; 39: 938-944.

32. Caldwell C, Sankreacha R, Lightstone AW et al. Contamination during a brachytherapy procedure. Health Phys 2007; 92 (2 Suppl): S8-S12.

33. Chang MY, Kamrava M, Demanes DJ et al. Intraoperative ultrasonography-guided positioning of iodine 125 plaque brachytherapy in the treatment of choroidal melanoma. Ophthalmology 2012; 119: 1073-1077.
34. Gagne NL, Leonard KL, Rivard MJ. Radiobiology for eye plaque brachytherapy and evaluation of implant duration and radionuclide choice using an objective function. Med Phys 2012; 39: 3332-3342.

35. Valentini V, Maurizi F, Tagliaferri L et al. Spider: managing clinical data of cancer patients treated through a multidisciplinary approach by a palm based system. Ital J Public Health JPH 2008; 6.

36. Tagliaferri L, Kovács G, Autorino R et al. ENT COBRA (Consortium for Brachytherapy Data Analysis): interdisciplinary standardized data collection system for head and neck patients treated with interventional radiotherapy (brachytherapy). J Contemp Brachytherapy 2016; 8: 336-343. 